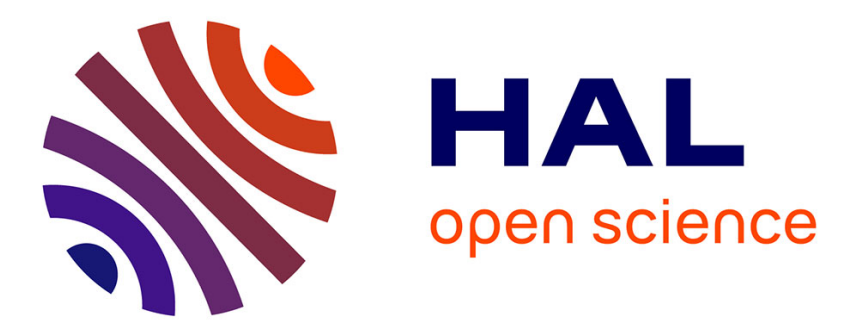

\title{
Influence du sexe et de l'âge des insectes vecteurs injectés dans l'épreuve d'infectivité des jaunisses des plantes. Mesure radiographique du volume injecté à Euscelidius variegatus (Kirschbaum) \\ Catherine Kuszala
}

\section{To cite this version:}

Catherine Kuszala. Influence du sexe et de l'âge des insectes vecteurs injectés dans l'épreuve d'infectivité des jaunisses des plantes. Mesure radiographique du volume injecté à Euscelidius variegatus (Kirschbaum). Agronomie, 1986, 6 (6), pp.591-598. hal-00884914

\author{
HAL Id: hal-00884914 \\ https://hal.science/hal-00884914
}

Submitted on 1 Jan 1986

HAL is a multi-disciplinary open access archive for the deposit and dissemination of scientific research documents, whether they are published or not. The documents may come from teaching and research institutions in France or abroad, or from public or private research centers.
L'archive ouverte pluridisciplinaire HAL, est destinée au dépôt et à la diffusion de documents scientifiques de niveau recherche, publiés ou non, émanant des établissements d'enseignement et de recherche français ou étrangers, des laboratoires publics ou privés. 


\section{Influence du sexe et de l'âge des insectes vec- teurs injectés dans l'épreuve d'infectivité des jaunisses des plantes. Mesure radiographique du volume injecté à Euscelidius variegatus (Kirschbaum)}

Catherine KUSZALA

I.N.R.A., Station de Recherches sur les Mycoplasmes et les Arbovirus des Plantes, BV 1540, F 21034 Dijon

En vue d'améliorer la sensibilité et la précision des épreuves d'infectivité, nous avons comparé la capacité des mâles et des femelles de la cicadelle Euscelidius variegatus (Kirschbaum) à transmettre la flavescence dorée de la vigne après microinjection.

Les résultats indiquent que, dans le cas étudié, la capacité vectrice des mâles injectés est bien supérieure à celle des femelles. Or, il est apparu que le volume de liquide reçu et retenu par les femelles et par les mâles est à peu près le même, bien que le poids des femelles soit plus élevé que celui des mâles. Il semblerait donc que l'inoculation d'une femelle ne conduise pas régulièrement à son infection, lorsque les ovaires et leurs dépendances sont atteints. Cette hypothèse semble confirmée par la très grande sensibilité des très jeunes femelles. Elle n'est pas en opposition avec les données bibliographiques concernant d'autres espèces.

D'autre part, le volume reçu et retenu par les mâles varie moins que celui des femelles. Il est même très stable chez les mâles qui ont plus de $5 \mathrm{j}$. Il est donc recommandé de n'inoculer que des mâles pour donner aux épreuves d'infectivité le maximum de fidélité. Ceci est particulièrement intéressant dans le cas d'E. variegatus où les mâles transmettent mieux que les femelles.

Le volume injecté aux mâles de cette espèce, mesuré à l'aide d'un radioisotope, est de 0,67 $\mu \mathrm{l}$. C'est ce volume que nous avons retenu pour évaluer les concentrations à partir des épreuves d'infectivité.

Mots clés additionnels : Cicadelles, flavescence dorée, vigne, microinjection du vecteur, test d'infectivité. Radiomeasurement of the volume injected into Euscelidius variegatus (Kirschbaum).

In order to improve the sensitivity and accuracy of infectivity tests, we compared the transmission efficiency of male and female leafhoppers (Euscelidius variegatus Kirschbaum) after injection with grapevine flavescence dorée. The results show that vector efficiency of injected males was higher than that of females (tables 1 and 2). This is surprising because the weight of females was always significantly greater than that of males (fig. 1 and 5). In fact, female weight chiefly rose on the 3rd day of adult life, when eggs are developing in their bodies (fig. 2). Consequently, the volume of solution received and retained by injected insects was nearly the same for males and females (fig. 3), but the variation was greater for females. We concluded that injection of a female did not regularly result in infection if it reached the ovaries and surrounding organs. This hypothesis seemed to be confirmed by the high receptivity of very young females, which did not differ from that of very young males. Moreover, this hypothesis was consistent with results obtained in comparing infection by feeding or injection for other leafhopper species reported in the literature. In addition, the volume injected and retained by males varied less than that of females and was very stable in males older than 5 days (fig. 4 and 5). Therefore, to ensure maximum reproducibility in infectivity tests, it was best to inoculate only males. This was especially important in the case of $E$. variegatus where the males were more efficient vectors than females. The volume injected in the males of this species was $0.67 \mu \mathrm{l}$ measured by means of a radioisotope, and we thereafter used this volume to determine concentrations in the infectivity assays.

Additional key words : Leafhoppers, flavescence dorée, grapevine, vector microinjection, infectivity test. 


\section{INTRODUCTION}

Les maladies des végétaux propagées par des arthropodes figurent parmi les plus graves en raison de leur caractère épidémique. Parmi elles, les jaunisses, longtemps définies sur des critères symptomatologiques seuls utilisables, sont particulièrement redoutables car elles se manifestent par un blocage de l'assimilat chlorophyllien qui n'alimente plus les organes de réserve, objet de la récolte.

Depuis 1967, les techniques de l'observation in situ sur des coupes ultrafines végétales ont permis de distinguer parmi les jaunisses celles qui sont provoquées par des virus et celles qui sont associées à la présence d'organismes procaryotes dépourvus de paroi. Ces dernières ont été appelées jaunisses à mycoplasmes ou à MLO (Mycoplasma-like-organisms), en raison de la ressemblance morphologique de ces procaryotes avec les mycoplasmes (DOI et al., 1967).

Les MLO se distinguent des mycoplasmes connus en pathologie animale par leur habitat intracellulaire et leur mode de transmission. Ils prolifèrent dans les tubes criblés de la plante qu'ils nécrosent, provoquant les symptômes de "jaunisses », et sont transmis de plante à plante par des insectes hémiptères, cicadelles ou psylles. Le MLO est prélevé par le vecteur au moyen de son canal alimentaire. Il gagne le tube digestif, pénètre la paroi intestinale, s'y multiplie et la traverse. Le MLO est ensuite porté passivement par l'hémolymphe vers les glandes salivaires et les autres organes. Les insectes sont alors capables de transmettre la maladie par le canal salivaire.

Les MLO sont très mal connus car ils ne sont pas cultivables in vitro et leur purification se heurte à leur hétérogénéité de taille, de forme et de densité.

La seule technique qui permette d'identifier ces agents pathogènes in vitro, et donc de faire quelques progrès dans leur purification, est l'épreuve d'infectivité par l'injection au vecteur. Il n'est pas possible en effet d'inoculer un jus infectieux à la plante ainsi qu'il est habituellement d'usage en virologie. On est obligé d'injecter l'extrait infectieux à des insectes sains vecteurs de la maladie et on révèle l'infectivité de ceux-ci en les plaçant sur des plantes saines. Cette technique est longue et nécessite beaucoup de matériel animal et végétal. Mais elle représente l'instrument unique de la première approche pour l'étude de ces pathogènes in vitro.

Notre laboratoire a proposé un moyen d'évaluer la concentration des extraits en fonction des proportions de plantes devenues malades (CAUDWELL, 1977). Ces évaluations, poursuivies pendant plusieurs années, ont révélé des fluctuations importantes dans les résultats des épreuves d'infectivité réalisées dans les mêmes conditions. Nous avons tenté de réduire ces variations en concentrant notre attention sur le poids des insectes et le volume injecté, selon le sexe, l'âge et la quantité d'œufs portés par les femelles.

\section{MATÉRIEL ET MÉTHODES}

Parmi les modèles dont nous disposons au laboratoire nous avons travaillé sur l'épreuve d'infectivité de la flavescence dorée de la vigne qui utilise Euscelidius variegatus (Kirschbaum), Homoptère Jassidae, comme vecteur de laboratoire et Vicia faba L. comme plante hôte. (CAUDWELl et al., 1972).

\section{A. La mesure de l'infectivité}

\section{Préparation des insectes sains à inoculer}

Les insectes sont élevés en salle climatisée selon une technique qui a été normalisée (CAUDWELL \& LARRUE, 1977) : un nouvel élevage est lancé toutes les 2 semaines et les cicadelles sont ainsi sériées par tranches d'âge de $14 \mathrm{j}$.

Pour les épreuves d'infectivité, nous injectons des cicadelles « jeunes », passées au stade adulte depuis moins de 2 semaines.

\section{L'inoculum infectieux}

Les élevages infectieux sont obtenus selon la méthode déjà décrite (CAUDWELL \& LARRUE, 1977). 36 cicadelles infectieuses sont broyées dans $6 \mathrm{ml}$ de milieu au moyen d'un Potter à main. Après clarification, l'extrait est passé au travers d'un préfiltre en fibre de verre, puis d'un filtre membrane à pores de $450 \mathrm{~nm}$. Cet extrait contient $10^{6}$ unités infectieuses par ml (CAUDWELL, 1977).

\section{La technique d'inoculation}

Les insectes sont immobilisés par le gaz carbonique ou l'éther sous un éclairage froid à fibres optiques. Leur inoculation est réalisée avec une pipette Pasteur étirée en capillaire très fin. Nous avons préféré ces pipettes aux microseringues car une partie du liquide injecté est rejeté par l'insecte. L'injection se fait dans le flanc de l'insecte entre les $4^{\mathrm{e}}$ et $5^{\mathrm{e}}$ segments, en biais, la pointe de la pipette dirigée vers la tête de l'insecte. La pipette Pasteur est reliée par un tube souple à la bouche de l'opérateur qui module ainsi la pression ; celle-ci est arrêtée au premier gonflement de l'insecte, qui s'accompagne d'un suintement de liquide au niveau de la piqûre. Une épreuve d'infectivité comprend normalement l'injection d'une trentaine d'insectes.

\section{Manipulation des insectes après l'injection}

Les 30 cicadelles injectées sont placées sur 2 plants de fèves pendant les $21 \mathrm{j}$ de la période de latence de l'insecte. Au bout de ce temps, les survivantes sont réparties à raison de 3 par plante sur de jeunes fèves saines où on les maintient pendant $7 \mathrm{j}$. Les proportions de ces plantes devenant malades permettent d'évaluer l'infectivité des extraits selon une formule déjà publiée (CAUDWELL, 1977).

\section{B. Evaluation du poids de l'insecte et du volume injecté}

\section{Préparation des insectes à injecter}

L'élevage des insectes est mené comme précédemment. Cependant, il est ici nécessaire de mieux préciser leur âge afin d'évaluer l'influence de la maturation 
des œufs chez les femelles. Cela est possible en prélevant quotidiennement parmi les larves du $5^{\text {e }}$ stade celles qui passent à l'état adulte. L'âge est alors compté à partir de ce jour. Avant leur injection, les insectes, endormis à l'éther, sont pesés individuellement sur une balance sensible au 1/100 de $\mathrm{mg}$.

\section{Mesure radiographique du volume de liquide injecté retenu par l'insecte}

Les méthodes utilisant une micro-seringue étalonnée ont l'inconvénient de mesurer le volume injecté et non le volume retenu par la cicadelle. Nous avons donc choisi de mesurer la radioactivité retenue par l'insecte lorsqu'on lui injecte une solution de radioactivité connue. L'injection se fait comme précédemment. Le radioisotope choisi est une solution de tyrosine radioactive marquée uniformément au niveau du carbone $\left(\mathrm{U}-{ }^{14} \mathrm{C}\right)$, (CEA : solution $\left.\mathrm{HCl} \mathrm{N} / 5000 \mathrm{pH} 4\right)$, avec une radioactivité spécifique légèrement supérieure à $5 \mu \mathrm{curies} / \mathrm{ml}$ de solution.

Les cicadelles injectées sont laissées en attente pendant $15 \mathrm{mn}$, le temps que s'écoule l'excédent de liquide. Elles sont ensuite anesthésiées au gaz carbonique et lavées 2 fois à l'alcool éthylique pour éliminer la part de solution de tyrosine rejetée par l'insecte.

Chaque cicadelle est mise individuellement dans une fiole à scintillation. L'insecte est disséqué dans $8 \mathrm{ml}$ de mélange scintillant Dynagel (J. T. Baker). C'est à ce moment que sont comptés les œufs matures. Les fragments sont ensuite homogénéisés aux ultra-sons pendant $1 \mathrm{mn}$ à $180 \mathrm{~V}$ (Sonicatum Annemasse type 150 TS $20 \mathrm{~K}$ ) dans de la glace avec une sonde moyenne. La quantité de l'élément radioactif présent dans chaque cicadelle est dosée avec un compteur à scintillation liquide (Beckman LS 7500).

Tous les résultats ont été éprouvés statistiquement à l'aide des épreuves $\chi^{2}$ et $\mathrm{F}$. Les calculs ont été faits sur ordinateur Mini 6 avec l'aide de A. CARTERON (I.N.R.A.-Dijon).

\section{RÉSULTATS}

\section{A. Influence du sexe des insectes injectés sur les résul- tats des épreuves d'infectivité}

Nous avons comparé les résultats des épreuves d'infectivité d'un même extrait injecté simultanément à des cicadelles mâles et femelles. Les insectes ont été ensuite répartis à raison de 3 par plante, en séparant sur des plantes différentes les mâles et les femelles. Nous présentons ici les résultats obtenus avec 12 extraits différents. Les proportions de plantes inoculées devenues malades, selon le sexe des insectes, sont données dans le tableau 1. Nous voyons que les mâles injectés ont donné de plus fortes proportions de plantes malades que les femelles. Ces résultats, régulièrement retrouvés, sont hautement significatifs $\left(\chi^{2}=8,59\right.$ : significatif au risque 1 p. 100). Nous avons calculé pour ces essais, selon la loi binomiale $\left(\mathrm{P}_{0}=\mathrm{qo}^{3}\right)$ (CAUDWELL, 1977), les proportions de cicadelles devenues infectieuses : 13 p. 100 seulement
TABLEAU 1

Infectivité des insectes injectés, selon leur sexe. Infectivity of injected insects, according to their sex.

\begin{tabular}{|c|c|c|}
\hline $\begin{array}{l}\text { Sexe des } \\
\text { insectes } \\
\text { injectés }\end{array}$ & $\begin{array}{l}\text { Proportions de } \\
\text { plantes infectées } \\
\text { (avec } 3 \text { cic./pl.) }\end{array}$ & $\begin{array}{c}\text { Proportions } \\
\text { calculées de } \\
\text { cicadelles infectées }\left({ }^{*}\right)\end{array}$ \\
\hline Femelles & $\begin{array}{c}29 / 85 \\
(34 \%)\end{array}$ & $\begin{array}{l}33 / 255 \\
(13 \%)\end{array}$ \\
\hline Mâles & $\begin{array}{c}47 / 83 \\
(57 \%)\end{array}$ & $\begin{array}{l}60 / 249 \\
(24 \%)\end{array}$ \\
\hline$\chi^{2}$ & $\begin{array}{c}8,59 \\
\text { (La différence est } \\
\text { significative au } \\
\text { risque } 1 \% \text { ) }\end{array}$ & \\
\hline
\end{tabular}

* Le calcul des proportions de cicadelles infectées a été fait selon la loi binomiale $\mathrm{Po}=\mathrm{qo}^{3}$ où Po est la probabilité pour qu'une plante ne soit pas infectée et qo la probabilité pour qu'une cicadelle ne soit pas infectieuse, avec 3 cicadelles par plante (CAUDwelL, 1977).

* The binomial law $\mathrm{Po}=\mathrm{qo}^{3}$ was used to calculate the proportion of infected leafhoppers. Po is the probability that a plant is not infected and qo is the probability that a leafhopper is not infectious, with 3 leafhoppers per plant (CAUDwEll, 1977).

des femelles se sont révélées infectieuses, contre 24 p. 100 des mâles (tabl. 1).

Il est possible de comparer également la période de latence chez les mâles et les femelles. Celle-ci est évaluée d'après les symptômes apparus sur les plantes qui ont porté les 15 cicadelles injectées pendant les $21 \mathrm{j}$ qui ont suivi leur inoculation. Nous dénombrons ainsi les cas où la période de latence est inférieure ou supérieure à $21 \mathrm{j}$. Le tableau 2 donne les résultats obtenus sur les mêmes échantillons que précédemment. Il apparaît clairement que les mâles injectés transmettent plus rapidement que les femelles. La différence est significative au seuil 2 p. 100.

\section{TABLEAU 2}

Temps d'incubation chez les mâles et les femelles, exprimé selon les infections obtenues sur des plants de fèves qui ont porté les cicadelles injectées pendant les $21 j$ qui ont suivi l'inoculation de l'insecte. Incubation time of males and females expressed as number of broad bean plants infected during the 21 days after insect inoculation.

\begin{tabular}{llll}
\hline & o infectés & 9 infectées & $\chi^{2}$ \\
\hline $\begin{array}{l}\text { Proportions de } \\
\text { plantes infectées }\left(^{*}\right)\end{array}$ & $16 / 28$ & $7 / 28$ & $5,98(* *)$ \\
\hline \hline
\end{tabular}

$\left(^{*}\right)$ Chacune des plantes a porté 15 cicadelles pendant les 3 semaines d'incubation.

(**) Différence significative au seuil $2 \%$.

L'ensemble de ces résultats permet d'admettre que, dans le cas étudié, la capacité vectrice des mâles injectés est très supérieure à celle des femelles (le double environ). Ces résultats ont été régulièrement retrouvés dans tous les essais que nous avons réalisés ultérieurement avec la même espèce de cicadelle, $E$. variegatus. 
Ils ont été confirmés par l'amélioration notable des résultats obtenus, lorsque nous avons décidé de n'inoculer que des mâles: les épreuves d'infectivité ont donné des résultats supérieurs et plus réguliers.

Cette meilleure performance des mâles injectés est $a$ priori surprenante car les femelles apparaissent nettement plus volumineuses que les mâles. Or la base même d'une épreuve d'infectivité est que son résultat est fonction du nombre d'unités infectieuses injectées et, par conséquent, du volume de solution infectieuse injecté. Il est donc nécessaire de savoir pourquoi les femelles, plus volumineuses que les mâles, sont moins performantes et donnent des résultats moins réguliers. Nous avons porté notre analyse sur le poids des insectes et sur le volume qui leur est injecté.

\section{B. Analyse du poids des mâles et des femelles}

L'analyse du poids des mâles et des femelles avant leur injection est réalisée sur plusieurs échantillons d'insectes jeunes passés à l'état adulte depuis 1 à $15 \mathrm{j}$. Les cicadelles sont anesthésiées à l'éther, puis pesées individuellement. Les résultats sont donnés dans la figure 1 .

Nous voyons immédiatement que les femelles sont plus lourdes que les mâles et ceci de façon très significative. Cette différence est d'ailleurs parfaitement perceptible à l'œil nu.

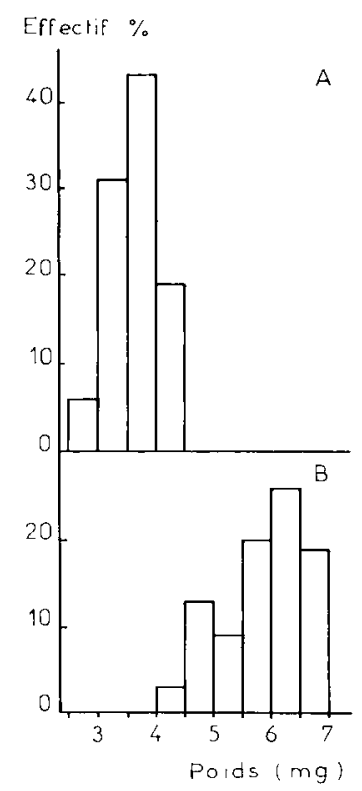

Figure 1

Poids comparé des cicadelles mâles (A) et femelles (B) sur des échantillons de $\mathrm{E}$. variegatus (adultes âgés de 1 à 15 jours).

A mâles : effectif 95 ; moyenne $3,64 \mathrm{mg}$ : variance 0,12 ; écart type 0,34.

$B$ femelles : effectif 137 ; moyenne $5,88 \mathrm{mg}$; variance 0,60 ; écart type 0,77 .

Les populations des mâles et des femelles sont significativement différentes en ce qui concerne le poids : test $f_{l, 231}=687,61$ (significatif au seuil 1 p. 100).

Weights of adult male $(A)$ and female (B) leafhoppers $E$. variegatus aged from 1 to 15 days.

A males : sample size 95 ; mean $3.64 \mathrm{mg}$; variance 0.12 ; standard deviation 0.34 .

$B$ females : sample size 137 ; mean $5.88 \mathrm{mg}$; variance 0.60 ; standard deviation 0.77 .

The body weights of male and female populations were significantly different at the $1 \%$ level of probability $\left(f_{1,231}=687.61\right)$.
En second lieu, la variance des poids dans la population des femelles est nettement supérieure à la variance des poids dans la population des mâles. Cette variabilité du poids des femelles pourrait provenir des variations dans le nombre des œufs qu'elles portent. Les pontes occupent en effet un volume très important dans leur abdomen.

Il est difficile d'établir une corrélation entre le poids de chaque femelle et l'évolution du nombre de ses œufs. Le nombre des œufs matures est en effet évalué sur l'insecte disséqué et ceci ne peut être fait qu'une fois. Il est par contre possible de considérer des populations d'insectes, séparées en tranches d'âges journalières, et d'étudier la relation entre le poids et le nombre des œufs.

La figure 2 donne le poids des insectes, mâles ou femelles, selon les tranches d'âges. Le poids des mâles et sa variance sont parfaitement constants, quel que soit l'âge ( $f_{6,94}=1,47$, non significatif). Le poids des femelles par contre varie beaucoup avec l'âge $\left(f_{7,136}=9,83\right.$ significatif au seuil 1 p. 100). On constate sur la figure 2 que les jeunes femelles (adultes depuis 1 à $3 \mathrm{j}$ ) sont moins lourdes que leurs aînées. Elles prennent brusquement du poids entre le $3^{\text {e }}$ et le $5^{\mathrm{e}} \mathrm{j}$ d'âge adulte.

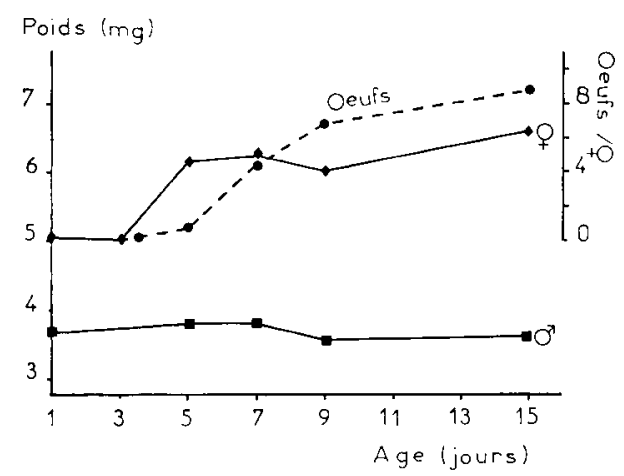

Figure 2

Variations du poids des insectes mâles ou femelles selon les tranches d'âge adulte (traits pleins), et variation parallèle du nombre des ceufs des femelles (trait discontinu).

Variations in male and female insect body weight according to age (solid lines) and number of eggs in the female (dotted line).

Il est facile de mettre ces variations du poids des femelles en relation avec le nombre d'œufs « matures » obtenus par la dissection de ces mêmes femelles (trait discontinu sur la figure 2). Le nombre moyen d'œufs matures récoltés augmente rapidement à partir du $5^{\mathrm{e}} \mathrm{j}$; le décalage entre les 2 courbes est probablement dû au temps de maturation des œufs.

Ces résultats sont apparus comme très constants dans tous nos élevages de cicadelles. Ils ont été reproduits de façon identifique dans 2 salles d'élevage différentes et en plusieurs saisons.

Nous allons voir maintenant si l'importance des œufs, révélée ici par les variations dans le poids des femelles, intervient dans le volume de liquide injecté et retenu par l'insecte. 


\section{Analyse du volume injecté chez les mâles et les femelles}

Nous avons vérifié, sur le même échantillonnage d'insectes, le volume injecté, soit aux mâles, soit aux femelles, par la méthode de l'injection d'une solution radioactive. Les résultats sont donnés dans la figure 3 .

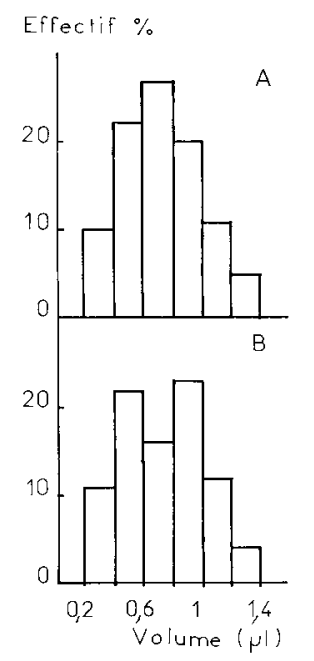

Figure 3

Comparaison du volume injecté aux mâles (A) et aux femelles (B) (adultes âgés de 1 à 15 jours).

A mâles: effectif 95 ; moyenne $0,73 \mu \mathrm{l}$; variance 0,07 ; écart type 0,26.

$B$ femelles : effectif 137 ; moyenne $0,8 \mu \mathrm{l}$; variance 0,16 ; écart type 0,40 .

Les populations des mâles et des femelles ne sont pas significativement différentes en ce qui concerne le volume injecté et retenu par l'insecte : $f_{I, 231}=3,21$ (non significatif), mais les variances sont différentes.

Comparison of the volumes injected in males $(A)$ and females $(B)$ adults aged from 1 to 15 days.

A males : sample size 95 ; mean $0.73 \mu \mathrm{l}$; variance 0.07 ; standard deviation 0.26 .

$B$ females : sample size 137 ; mean $0.8 \mu \mathrm{l}$; variance 0.16 ; standard deviation 0.4

The male and female populations did not differ significantly in the average amount of injected liquid retained by the insect $\left(f_{1,23 l}=3.21\right)$, but the variances were different .

Il apparaît immédiatement que les volumes injectés aux mâles et aux femelles sont voisins, ils ne sont pas statistiquement différents. Ce résultat pourrait surprendre puisque nous avons vu que le poids des femelles est bien supérieur au poids des mâles, mais il peut s'expliquer par l'importance du volume occupé par les œufs dans l'abdomen des femelles. En second lieu, nous remarquons que la variance chez les femelles est supérieure à celle des mâles. La distribution prend même un aspect bimodal. C'est là un fait important qui a été retrouvé sur tous les autres échantillons de femelles.

Il s'est révélé intéressant, ici encore, de séparer les insectes par tranches d'âges (fig. 4). Le volume injecté, soit aux mâles, soit aux femelles, n'est pas constant selon les tranches d'âge $\left(f_{6,94}=6,75\right.$, chez les mâles ; $\mathrm{f}_{7,136}=15,64$ chez les femelles : les 2 sont significatifs au seuil 1 p. 100). Il apparaît clairement sur ces graphiques que l'essentiel de cette hétérogénéité provient des très jeunes insectes (moins de $5 \mathrm{j}$

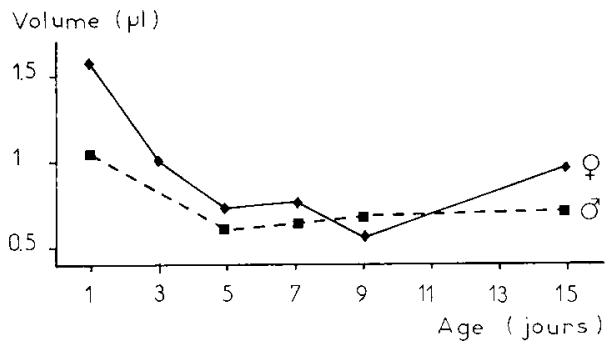

Figure 4

Volume injecté et retenu par l'insecte selon le sexe et la tranche d'âge.

Volume injected and retained by the adult insect according to sex and age group.

d'âge adulte) chez lesquels le volume injecté est très important et d'autant plus important que l'insecte est plus jeune.

L'hypothèse la plus simple pour expliquer ce fait serait que les insectes très jeunes, à cuticule souple, seraient en quelque sorte extensibles. Ils le seraient d'autant plus que la sclérotinisation de la cuticule est plus faible et qu'ils sont plus jeunes. Ceci rend bien compte de la diminution rapide de ce phénomène entre le $1^{\text {er }}$ et le $5^{\mathrm{e}} \mathrm{j}$.

La diminution du volume injecté entre le $1^{\text {er }}$ et le $5^{\mathrm{e}} \mathrm{j}$ est apparente chez les mâles comme chez les femelles, mais elle est significativement plus importante chez les femelles. La sclérotinisation de la cuticule se superpose chez ces dernières à l'apparition progressive des œufs, qui survient justement entre le $3^{\text {e }}$ et le $5^{\mathrm{e}} \mathrm{j}$ comme nous l'avons vu précédemment (fig. 2). L'apparition des œufs contribue à réduire peu à peu le " volume libre » de l'abdomen de la femelle jusqu'à un volume d'équilibre qui est celui de la femelle pondeuse, voisin de celui des mâles (fig. 4).

Nous retiendrons que le volume injecté, peu différent chez les mâles et chez les femelles, subit moins de

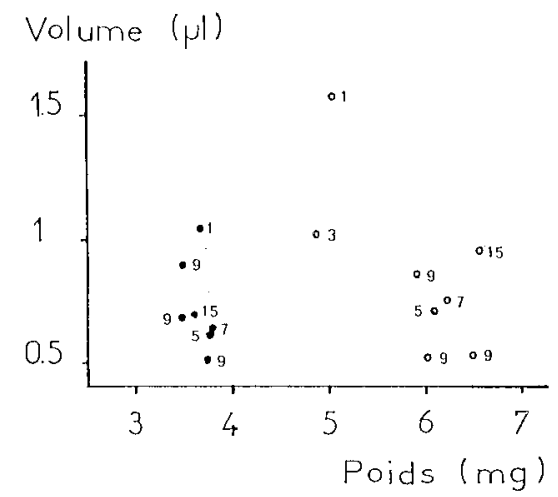

Figure 5

Relation entre le poids des insectes et le volume injecté.

Les nombres à côté des points indiquent l'âge adulte des insectes. Les mâles et les femelles forment 2 populations distinctes en ce qui concerne le poids. Les insectes jeunes (inf. à $5 \mathrm{j}$ ) se distinguent par le grand volume qui peut leur être injecté.

- = mâles $\quad 0=$ femelles

Relationship of insect weight and injected volume.

The numbers next to the points indicate the age of the adult insect. Males and females formed two distinct populations with regard to weight. In very young insects (less than 5 days) a great volume can be injected.

- = males $\quad 0=$ females 
fluctuations chez les mâles que chez les femelles. D'autre part, on a intérêt dans les épreuves d'infectivité, à ne pas utiliser les très jeunes adultes qui sont extensibles, du fait de leur cuticule souple. Nous tiendrons compte de ces éléments pour définir dans nos conclusions les insectes qui sont à injecter ainsi que le volume inoculé qui est à prendre en considération pour le calcul du résultat des épreuves d'infectivité.

Pour résumer ces 2 chapitres concernant les poids et les volumes, nous donnons dans la figure 5 les relations qui existent, au sein d'une population de cicadelles, entre le poids des insectes et le volume injecté. Deux éléments apparaissent nettement :

- en ce qui concerne le poids, les mâles et les femelles constituent 2 populations distinctes ;

- du point de vue du volume injecté, les très jeunes insectes s'individualisent (les adultes âgés de 1 à $3 \mathrm{j}$ et particulièrement les jeunes femelles d'un jour).

\section{DISCUSSION ET CONCLUSION}

Cette étude apporte un certain nombre d'éléments au problème de la sensibilité et de la fidélité de l'épreuve d'infectivité par la micro-injection des cicadelles.

A. Le premier fait à retenir est que, dans le cas étudié, la capacité vectrice des mâles injectés est bien supérieure à celle des femelles (tabl. 1 et 2).

Ce résultat semble paradoxal : les femelles, plus lourdes et plus volumineuses que les mâles (fig. 1) et ceci à tout âge (fig. 2), devraient recevoir plus d'agents infectieux.

Or, il est apparu que le volume retenu par les femelles est peu différent de celui qui est retenu par les mâles (fig. 3). Le volume interne des femelles est en effet considérablement réduit par la présence des œufs. Selon le principe de l'épreuve d'infectivité, on pourrait alors penser que la capacité vectrice des mâles et des femelles injectés est sensiblement égale. Or il n'en est rien, puisque les mâles ont une meilleure aptitude à la transmission que les femelles et une meilleure régularité. Le volume injecté aux femelles ne conduit donc pas toujours à l'infection de l'insecte.

Nous avons pensé que l'inoculation d'une femelle ne conduit pas régulièrement à son infection lorsque les ovaires et leurs dépendances sont atteints. Ces organes volumineux sont en effet parfaitement isolés, pour ce qui concerne les MLO, du reste du corps de la femelle puisqu'il n'y a jamais de transmission transovarienne des MLO. Or ils se trouvent exactement au point où se font les injections. Le caractère aléatoire de l'injection au niveau des ovaires expliquerait bien la moindre sensibilité moyenne des femelles et la plus grande variabilité de leurs performances (fig. 3 et 4).

Nous avons tenté d'étayer cette hypothèse en vérifiant si les femelles n'ayant pas encore ces organes développés sont aussi sensibles que les mâles, voire plus « sensibles » puisque le volume injecté est plus grand (fig. 4). L'expérience a confirmé cette hypothèse en montrant la très grande sensibilité des très jeunes femelles. Cette expérience est cependant difficile à mener sur une grande échelle en raison de leur fragilité ; elles meurent souvent après l'injection. Nous avons de même tenté d'injecter des larves de $5^{\mathrm{e}}$ stade. La transmission a été aussi bonne par les mâles que par les femelles. Mais ici encore l'expérience est difficile à réaliser car ces larves sont plus petites et encore plus fragiles que les jeunes adultes. On ne peut donc conseiller d'injecter les larves, d'autant plus que le «volume » injecté doit être encore plus aléatoire que chez les jeunes adultes.

Nous sommes ainsi amenée à recommander de n'inoculer que les mâles dans les épreuves d'infectivité qui utilisent $E$. variegatus. Ceci est valable pour l'agent pathogène de la flavescence dorée, pour celui de la jaunisse PHY et, probablement, pour tous ceux qui sont transmis par cette espèce de cicadelle.

L'importance de l'isolement microbiologique des ovaires vis-à-vis des MLO, soulignée pour expliquer la moindre sensibilité des femelles par injection et la plus grande variabilité du volume injecté, ferait supposer que ceci est valable pour toutes les espèces de vecteurs de MLO. Or, un certain nombre d'auteurs ont comparé la sensibilité respective des cicadelles mâles et femelles d'autres espèces lorsqu'elles sont infectées soit par injection, soit par ingestion.

Les femelles de la cicadelle Macrosteles fascifrons Stal., infectées par ingestion, transmettent mieux que les mâles les agents pathogènes de l'aster yellow (SWENSON, 1971), de la phyllodie du trèfle (CHIYKOWSKI, 1967) et de la prolifération du trèfle (CHIYKOWSKI \& SinHA, 1970) (tabl. 3). Deux hypothèses ont été émises pour expliquer ce fait. La $1^{\text {re }}$ suggère que les femelles dépensent plus de temps à leur nutrition pour compenser la demande provoquée par le développement des ovaires (CHIYKOWSKI, 1967 ; NAITO, 1965). Cette hypothèse devrait concerner toutes les espèces. Or, chez E. variegatus, ce sont les mâles infectés par nutrition qui transmettent mieux que les femelles (CAUDWELl \& LARRUE, comm. per.). Cette hypothèse ne peut donc être retenue.

Une $2^{\mathrm{e}}$ hypothèse a été émise pour expliquer la meilleure transmission des femelles de $M$. fascifrons infectées par ingestion. PURCELL (1982) invoque une meilleure perméabilité de l'intestin des femelles. Cette hypothèse émise depuis fort longtemps (STOREY, 1933), peut être vérifiée en infectant les insectes par injection. Ainsi, la barrière intestinale étant dépassée, les 2 sexes devraient devenir également infectieux. Chez les insectes injectés par l'agent de la phyllodie du trèfle, les mâles et les femelles deviennent également infectieux (SinHA \& CHIYKOWSKI, 1968). Mais ce n'est plus exact lorsque l'essai est fait avec l'agent de la prolifération du trèfle en utilisant pourtant la même cicadelle. Dans ce cas, les femelles restent plus infectieuses que les mâles (CHIYKOWSKI \& SINHA, 1970), (tabl. 3). Ce n'est pas vérifié non plus dans le cas de notre présent travail sur $E$. variegatus (où ce sont les mâles qui sont les plus infectieux, tabl. 1), bien que le volume injecté aux mâles et aux femelles soit à peu près égal (fig. 3).

En fait, nous constatons plutôt une tendance à un parallélisme dans l'aptitude à la transmission après injection ou après ingestion. D'une façon générale :

- lorsque les femelles sont plus sensibles après ingestion, elles le sont également après injection (cas de $M$. fascifrons avec la prolifération du trèfle) ; 
- lorsque les femelles sont moins sensibles après ingestion, elles le sont aussi après injection (cas de E. variegatus, avec la flavescence dorée et avec la jaunisse PHY). Jamais nous n'observons d'inversion de sensibilité (tabl. 3).

Ceci implique que s'il y a une différence de perméabilité (système de défense ?) au niveau de l'intestin, elle existe également, et dans le même sens, au niveau des autres organes qui sont infectés ultérieurement (corps gras, glandes salivaires, etc...). Si l'on admet que la voie normale d'infection des glandes salivaires est le transport passif des MLO par l'hémolymphe entre l'intestin et ces glandes, la différence de sensibilité entre mâles et femelles ne pourrait se situer, outre l'intestin, qu'au niveau des glandes salivaires.

En second lieu, nous percevons toujours plus ou moins nettement une diminution de l'infectivité des femelles par rapport aux mâles, lorsqu'elles sont infectées par injection au lieu de l'être par ingestion. Ceci se remarque chez $M$. fascifrons pour la phyllodie du trèfle, et également chez $E$. variegatus pour la flavescence dorée (tabl. 3). Notre présent travail souligne en outre le caractère aléatoire du résultat de l'injection des femelles (fluctuations dans le volume injecté). Nous avons relié ces faits à 2 raisons au moins :

- les femelles sont souvent injectées au niveau des ovaires, qui sont imperméables aux MLO,

- le volume injecté dans les femelles varie en fonction de la quantité des œufs qu'elles contiennent.
Comme nous l'avons déjà dit, il y a donc toujours intérêt à n'utiliser que les mâles pour profiter des résultats les plus précis.

B. Le $2^{\mathrm{e}}$ point que nous voudrions souligner concerne l'âge des mâles à injecter. Nous utilisons normalement des mâles jeunes qui viennent de passer leur mue imaginale. Ceci permet d'utiliser la totalité de leur vie adulte pour le temps de latence et la transmission. Cependant, l'utilisation de mâles trop jeunes, adultes depuis moins de $5 \mathrm{j}$, introduit, nous l'avons vu, une nouvelle cause de variation. Ces jeunes mâles sont en effet capables de retenir un volume injecté près de 2 fois supérieur au volume retenu normalement par le mâle adulte (fig. 4). Nous devons donc utiliser des mâles jeunes, passés à l'état adulte depuis plus de $4 \mathrm{j}$.

C. Le dernier point que nous voudrions aborder concerne la détermination du volume injecté aux insectes. C'est une question qui a intéressé le monde scientifique et médical lorsqu'il s'agissait de vérifier s'il y avait ou non multiplication de certains « virus », sensu lato dans l'insecte vecteur (BLACK, 1953; MARAMOROSCH, 1956 ; MUELLER \& ROCHOW, 1961 ; Musil, 1964 ; Proeseler, 1964 ; etc...). Tous ces travaux ont utilisé la mesure du volume injecté à l'aide de microseringues graduées, qui ont l'inconvénient de mesurer le volume injecté et non le volume

TABLEAU 3

Sensibilité comparée des cicadelles mâles et femelles, selon qu'elles sont infectées par injection ou par ingestion. Sensitivity of male and female leafhoppers infected by injection or ingestion of the disease agent.

Vecteurs Aster Yellow

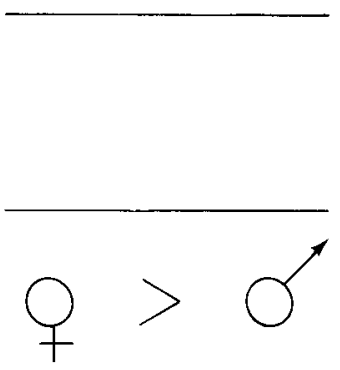

(c)
Macrosteles

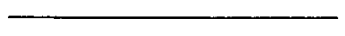

Phyllodie du trèfle

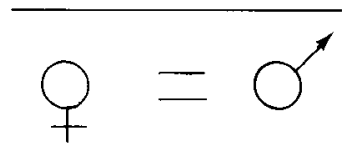

(a)

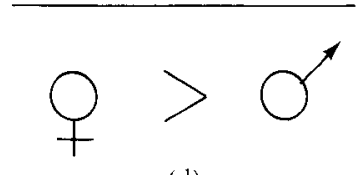

(d)

Euscelidius

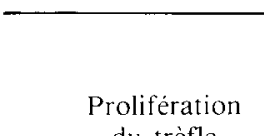

du trèfle

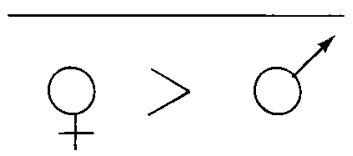

(b)

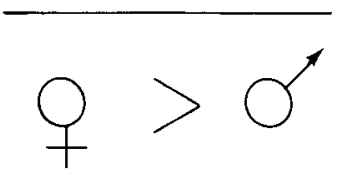

(b)

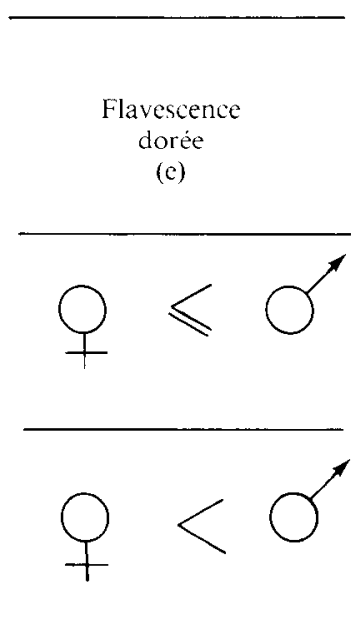

La comparaison est faite sur 2 espèces de cicadelles et sur 4 maladies. On voit qu'il y a un certain parallélisme entre la sensibilité par injection et par ingestion chez les 2 sexes, dans chaque cas. Cependant les femelles semblent d'une façon générale perdre de leur sensibilité par rapport aux mâles lorsqu'elles sont infectées par injection qui est souvent pratiquée au niveau des ovaires.

(a) Sinha \& ChIYkowski (1968); (b) ChIYkowski \& Sinha (1970); (c) Swenson (1971); (d) ChIykowski (1967); (e) Présent travail.

Comparison made with 2 leafhopper species and 4 diseases. There is an evident relationship between sensitivity by injection or ingestion for the 2 sexes. The females generally seem to lose their sensitivity compared to males when they are infected by injection. We explain this difference by the fact that females are often injected in the ovary region which does not lead regularly to infection. 
effectivement retenu par le vecteur. Nous avons introduit pour la mesure du volume injecté une nouvelle méthode basée sur la radioactivité de l'insecte après son injection avec un liquide de radioactivité connue. Cette technique a l'avantage de ne prendre en compte que le liquide retenu par l'insecte. Selon nos résultats, le volume moyen injecté en moyenne à un insecte mâle est de $0,73 \mu \mathrm{l}$ (fig. 3). Lorsqu'on élimine les mâles de moins de $5 \mathrm{j}$ de notre calcul, le volume moyen tombe à $0,67 \mu \mathrm{l}$. C'est ce volume que nous avons décidé de retenir pour la cicadelle $E$. variegatus.

Ces études ont finalement permis d'améliorer la précision et la sensibilité de l'épreuve d'infectivité par l'injection du vecteur. Elles ont ainsi contribué à la mise au point des méthodes d'extraction, de purification et de concentration de l'agent pathogène des jaunisses (MLO) dans notre laboratoire.

Reçu le 4 novembre 1985. Accepté le 7 mars 1986.

\section{REMERCIEMENTS}

Nous remercions A. CAudwell pour l'aide qu'il nous a apportée à divers stades de ce travail et, en particulier, pour ses suggestions et ses critiques au cours de la rédaction du manuscrit ainsi que M. Dalebroux et $\mathrm{A}$. Carteron pour leur contribution à l'étude statistique.

\section{RÉFÉRENCES BIBLIOGRAPHIQUES}

Black L. M., 1953. Viruses that reproduce in plants and insects. Ann. N. Y. Acad. Sci., 56 (3) 398-413.

Caudwell A., 1977. Aspects statistiques des épreuves d'infectivité chez les jaunisses (Yellow's) des plantes et chez les viroses transmises selon le mode persistant. Intérêt de la fève (Vicia faba) comme plante-test pour les jaunisses. Ann. Phytopathol., 9, 141-159.

Caudwell A., Larrue J., 1977. La production de cicadelles saines et infectieuses pour les épreuves d'infectivité chez les jaunisses à mollicutes des végétaux. L'élevage de Euscelidius variegatus KBM et la ponte sur mousse de polyuréthane. Ann. Zool. Ecol. anim., 9 (3), 443-456.

Candwell A., Kuszala C., Larrue J., Bachelier J. C., 1972. Transmission de la flavescence dorée de la fève à la fève par cicadelles des genres Euscelis et Euscelidius ; intervention possible de ces insectes dans l'épidémiologie du bois noir en Bourgogne. Ann. Phytopathol. $\mathrm{n}^{\circ}$ hors série, 181-189.

Chiykowski L. N., 1967. Some factors affecting the acquisition of clover phyllody virus by the aster leafhopper. J. econ. Entomol., 60, 849-853.

Chiykowski L. N., Sinha R. C., 1970. Sex and age of Macrosteles fascifrons in relation to the transmission of the clover proliferation causal agent. Ann. entomol. Soc. Am., 63, 1614-1617.

Doi Y., Teranaka M., Yora K., Asuyama H., 1967. Mycoplasma or PLT group-like microorganisms found in the phloem elements of plants infected with mulberry dwarf, potato witches' broom, aster yellows, or paulownia witches' broom. Ann. Phytopathol. Soc. Jap., 33, 259-266.
Maramorosch K., 1956. Semiautomatic equipment for injecting
insects with measured amounts of liquids containing viruses or toxic substances. Phytopathology, 46, 188-190.

Mueller W. C., Rochow W. F., 1961. An aphid injection method for the transmission of barley yellow dwarf virus. Virology, 14, 253-258.

Musil M., 1964. Multiplication of yellows-type plant viruses in Euscelis plebejus (Fallen) leafhoppers. Acta Virol., 8, 230-238.

Naito A., 1965. On relation between the feeding behavior of $\mathrm{LaO}$ delphax striatellus Fallen (Delphacidae) and the ovarial maturation. Jap. appl. Entomol. Zool., 9, 305-306.

Proeseler G., 1964. Der Nachweis der Vermehrung des Rübenkräusel Krankheitsvirus in Piesma quadrata (Fieb) mit Hilfe der Injektionstechnik. Naturwissenschaften, 6, 150-151.

Purcell A. H., 1982. Insect vector relationships with procaryotic plant pathogens. Annu. Rev. Phytopathol., 20, 397-417.

Sinha R. C., Chiykowski L. N., 1968. Distribution of clover phyllody virus in the leafhopper Macrosteles fascifrons (Stal). Acta Virol., 12, 546-550.

Storey H. H., 1933. Investigations of the mechanism of the transmission of plant viruses by insect vectors. Proc. $R$. Soc. Lond., Sér., B, 113, 463.

Swenson K. G., 1971. Relation of age, sex and mating of Macrosteles fascifrons to transmission of aster yellows. Phytopathology, 61, 657-659. 